\title{
Kurznachrichten aus der ÖFEB
}

Online publiziert: 18. Januar 2021

(c) Springer Fachmedien Wiesbaden GmbH, ein Teil von Springer Nature 2021

\section{1 ÖFEB Kongress 2021 vorschoben}

Da aufgrund der Corona Pandemie nicht abschätzbar ist, ob eine Großveranstaltung im Herbst durchführbar ist bzw. mit welchen Auflagen dabei zu rechnen ist, hat der Vorstand der ÖFEB beschlossen, den Kongress 2021 auf Herbst 2022 zu verschieben. Außerdem wird der Kongress nicht wie bisher geplant in Wien, sondern in Graz stattfinden. Herzlichen Dank an die Rektorate der PH Steiermark und der KPH Graz, dass sie sich bereiterklärt haben, den Kongress gemeinsam auszurichten.

Um auch 2021 im Austausch zu bleiben, plant der Vorstand eine Online Diskussionsreihe zu aktuellen bildungspolitischen Themen.

\section{Stellungnahme: Deutschförderklassen}

Die Österreichische Gesellschaft für Forschung und Entwicklung im Bildungswesen hat bereits in einer Stellungnahme im November 2019 darauf hingewiesen, dass organisatorisch von der Stammklasse getrennte „Deutschförderklassen“ kein adäquates Modell sind, um die Bildungsbenachteiligung von Schüler*innen, die bei Schuleintritt die Unterrichtssprache erst erwerben müssen, zu reduzieren. Nun liegen Untersuchungsergebnisse der Universität Wien (Schwab 2020) zur Praxis der seit 2018/19 bestehenden Deutschförderklassen vor. Sie dokumentieren vielfältige Probleme im Alltag der Deutschförderklassen. Das hat die ÖFEB zum Anlass genommen erneut eine Neuorganisation der Deutschförderung zu fordern. In der Stellungnahme werden Ergebnisse wissenschaftlicher Studien zur Deutschförderung aus linguistischer und pädagogischer Perspektive dargestellt. Die Ergebnisse belegen eindeutig, dass segregierte Förderung zu schlechteren Lernergebnissen führt als inklusive Modelle. Als Alternative wird die Umsetzung von inklusiven Modellen, die die Mehrsprachigkeit der Schüler*innen fördern, vorgeschlagen. Die vollstän- 
dige Stellungnahme finden Sie auf unserer Homepage unter: https://www.oefeb.at/ seiten/display/stellungnahmen

\section{3 ÖFEB und die Corona-Pandemie}

Covid 19 stellt Bildungsinstitutionen, Lehrende und Forscher*innen im Bildungsbereich oft vor schwierige Herausforderungen.

Bisher liegen kaum Erfahrungen und belastbare Befunde vor, die Hinweise auf die Steuerung und Gestaltung von Bildungs-und Forschungsprozessen unter diesen besonderen Bedingungen geben. Seit dem Frühjahr sind aber zahlreiche Studien und Publikationen erarbeitet worden mit dem Ziel, Hinweise auf Maßnahmen zur Gestaltung von Bildungsprozessen in der Pandemie und Informationen zu neuen Rahmenbedingungen für Lehre und Forschung zu geben oder die Auswirkungen der Pandemie auf verschiedene Gruppen zu untersuchen.

Eine Arbeitsgruppe aus dem Vorstand der ÖFEB hat in Kooperation mit der Sektion Medienpädagogik eine - nach 4 Kategorien geordnete - Linksammlung zusammengestellt, die vielleicht zur Orientierung in dieser schwierigen Zeit beitragen kann: https://www.oefeb.at/news/view/14.

Sie finden unter der Kategorie:

1. Lehren und Lernen in der Pandemie (E-Learning, Distance \& Home Learning: Erfahrungen und Qualitätskriterien) links zu Publikationen die Hinweise auf eine förderliche Gestaltung von Lernsituationen im Kontext von Corona geben.

Redaktion: Alessandro Barberi

2. Unter der Kategorie: Auswirkungen und Folgen der COVID-19-Pandemie für Bildung und Gesellschaft finden Sie Analysen und Stellungnahmen, die sich vor allem mit der Frage befassen

- mit welchen Veränderungen COVID-19 im Bereich von Bildung und Gesellschaft einhergeht

- und welche Implikationen sich daraus für verschiedene Sektoren des Bildungswesens ergeben.

Redaktion: Ferdinand Eder

3. Forschung in Zeiten von Corona bietet einerseits eine Zusammenstellung von links zu methodologischen Fragen der Forschung unter den spezifischen Rahmenbedingungen einer Pandemie und andererseits links zu empirischen Studien zu diesem Thema.

Redaktion: Christoph Helm

4. Unter Nationale und internationale Informationen (Aktuelles und Infos) finden Sie links zu aktuellen allgemeinen Informationen verschiedener Institutionen.

Redaktion: Matthias Huber 
Die Zusammenstellung stellt keinen Anspruch auf Vollständigkeit. Die verlinkten Studien - die im Einzelnen noch keiner Qualitätskontrolle unterzogen werden konnten - sollen eine erste Orientierung ermöglichen.

Wir möchten die Seite auf unserer Homepage laufend ergänzen und freuen uns über Hinweise, die wir gerne aufnehmen. Die Hinweise finden Sie auf unserer Homepage unter: https://www.oefeb.at/news/view/14

Katharina Soukup-Altrichter

Vorsitzende der ÖFEB 